\title{
Diversity of Online Community Activities
}

\author{
Tad Hogg \\ HP Labs \\ Palo Alto, CA 94304 \\ Gabor Szabo \\ HP Labs \\ Palo Alto, CA 94304
}

November 23, 2018

\begin{abstract}
Web sites where users create and rate content as well as form networks with other users display long-tailed distributions in many aspects of behavior. Using behavior on one such community site, Essembly, we propose and evaluate plausible mechanisms to explain these behaviors. Unlike purely descriptive models, these mechanisms rely on user behaviors based on information available locally to each user. For Essembly, we find the long-tails arise from large differences among user activity rates and qualities of the rated content, as well as the extensive variability in the time users devote to the site. We show that the models not only explain overall behavior but also allow estimating the quality of content from their early behaviors.
\end{abstract}

\section{Introduction}

Participatory web sites facilitate their users creating, rating and sharing content. Examples include Digg[.com] for news stories, Flickr[.com] for photos and Wikipedia[.org] for encyclopedia articles. To aid users in finding content, many such sites employ collaborative filtering [19] to allow users to specify links to other users whose content or ratings are particularly relevant. These links can involve either people who already know each other (e.g., friends) or people who discover their common interests through participating in the web site. In addition to helping identify relevant content, the resulting networks enable users to find others with similar interests and establish trust in recommendations [13].

The availability of activity records from these sites has led to numerous studies of user behavior and the networks they create. Observed commonalities in these systems suggest general generative processes leading to these observations. Examples include preferential attachment in forming networks and multiplicative processes leading to wide variation in user activity. While such models provide a broad understanding of the observations, they often lack causal connection with plausible user behaviors based on user preferences and the information available to users in making their decisions [29, 5]. Moreover, observed behavior can arise from a variety of mechanisms [23].

For predicting consequences of alternate designs of the web site, models including causal behavior are necessary. Establishing such models is more difficult than simply observing behavior: due to the possibility of confounding factors in observations, many different causal models can produce the same observations. Instead, such models would ideally use intervention studies and randomized trials to identify important causal relationships. In contrast to the wide availability of observational data on user behavior, such intervention studies are difficult, though this is situation is improving with the increasing feasibility of experiments in large virtual communities [4] and large-scale web-based experiments [27.

Nevertheless, identifying information readily available to users on a participatory web site can suggest plausible causal mechanisms. Such models provide specific hypotheses to test with future intervention experiments and also suggest improvements to overall system behavior by altering the user experience, e.g., available information or incentives. The simplest such approach considers average behavior of users on a site [21]. Such models can indicate how system behavior relates to the average decisions of many users. By design, such models do not address a prominent aspect of observed online networks: the long tails in their distributions of links and activity. Models including this diversity could be useful to improve effec- 
tiveness of the web sites by allowing focus on significantly active users or especially interesting content, and enhancing user experience by leveraging the long tail in niche demand [3].

A key question with respect to the observed diversity is whether users, content and the networks are reasonably viewed as behaviors arising from a statistically homogeneous population, and hence wellcharacterized by a mean and variance. Or is diversity of intrinsic characteristics among participants the dominant cause of the observed wide variation in behaviors? In the latter case, can these characteristics be estimated (quickly) from (a few) observations of behavior, allowing site design to use estimates of these characteristics, e.g., to highlight especially interesting content? Moreover, to the extent user diversity is important, what is a minimal characterization of this user variation sufficient to produce the observed long-tail distributions?

This paper considers these questions in the context of a politically-oriented web community, Essembly 1 . Unlike most such sites, Essembly provides multiple networks with differing nominal semantics, which is useful for distinguishing among some models. We consider plausible mechanisms users could be following to produce the observed long-tail behaviors both in their online activities and network characteristics. In the remainder of this paper, we first describe Essembly and our data set in Sec. 2. We then separately examine highly variable behaviors for users, content rating and network formation, in Secs. 3, 4, and 5. respectively. We suggest models to describe the observed characteristics of users, content, and the network, and consider their possible use during operation of the web site by helping identify user and content parameters early in their history. Finally we discuss implications and extensions to other participatory web sites.

In the three sections focusing on user behavior, resolve characteristics, and network structure, respectively, we first introduce the observations, then present a model describing these observations (subsections Model), and finally analyze the model parameters and predictions (subsections Behavior).

\section{Essembly}

Essembly is an online service helping users engage in political discussion through creating and voting on

\footnotetext{
${ }^{1}$ Essembly LLC at www.essembly.com
}

resolves reflecting controversial issues. Essembly provides three distinct networks for users: a social network, an ideological preference network, and an antipreference network, called friends (those who know each other in person), allies (those who share similar ideologies) and nemeses (those who have opposing ideologies), respectively.

The distinct social and ideological networks enable users to distinguish between people they know personally and people encountered on the site with whom they tend to agree or disagree. Network links are formed by invitation only and each link must be approved by the invitee. Thus all three networks in Essembly are explicitly created by users. Essembly provides a ranked list of ideologically most similar or dissimilar users based on voting history, thus users can identify potential allies or nemeses by comparing profiles. With regards to voting activity, the Essembly user interface presents several options for users to discover new resolves, for instance based on votes by network neighbors, recency, overall popularity, and degree of controversy.

Our data set consists of anonymized voting records for Essembly between its inception in August 2005 and December 2006, and the users and the links they have at that time in the three networks at the end of this period. Our data set has 15, 424 users. Essembly presents 10 resolves during the user registration process to establish an initial ideological profile used to facilitate users finding others with similar or different political views. To focus on user-created content, we consider the remaining 24,953 resolves, with a total of 1.3 million votes.

\section{$3 \quad$ Users}

Fig. 1 shows most users are active for only a short time (less than a day), as measured by the time between their first and last votes (this includes votes on the initial resolves during registration - users need not vote on all of them immediately). The 4762 users active for at least a day account for most of the votes and links, and we focus on these active users for our model. For these users, Fig. 1 shows an exponential fit to the activity distribution for intermediate times. Thus users who have sufficient interest in the system to participate for at least a few days behave approximately as if they decide to stop participating as a Poisson process. The additional decrease at long times (above 200 days or so) is due to the finite length 


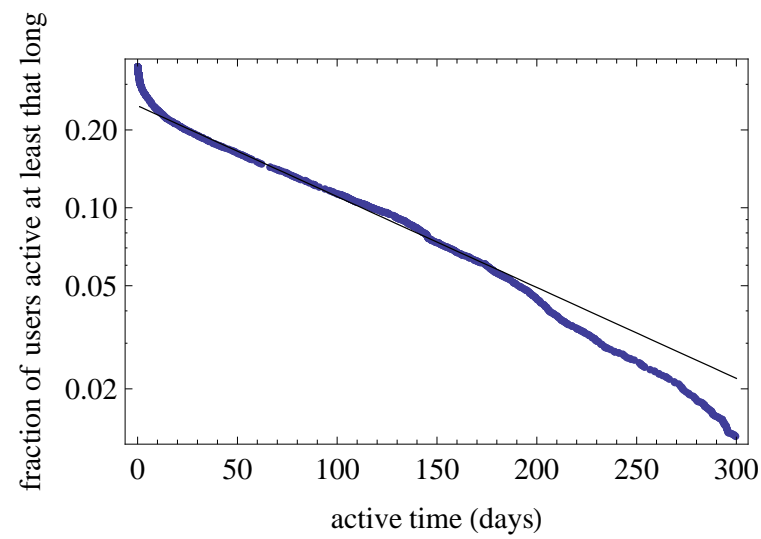

Figure 1: Distribution of activity times for users. The line shows an exponential fit to the values between 10 and 200 days, proportional to $e^{-t / \tau}$ where $\tau=$ 124 days.

of our data sample (about 500 days).

About a fifth of users have no votes on noninitial resolves. For the rest of the users, Fig. 2 shows the distribution of votes among users who voted at least once for noninitial resolves. These votes are close to a Zipf distribution in number of votes, with number of users with $v$ votes proportional to $v^{-\nu-1}$. The parameter estimates and confidence intervals in this and the other figures are maximum likelihood estimates [24, 17] assuming independent samples. This wide variation in user activity also occurs in other participatory web sites such as Digg [22].

The distribution of number votes per user arises from two factors: how long users participate before becoming inactive, and how often they vote while active.

\subsection{Model}

Fig. 3 summarizes our model for user behavior. This models the participation of users and their activities on the site while they are active. New users arrive in the system when they register, and we model this as a Poisson process with rate $\alpha$, and such users leave the system (i.e., become inactive) with a rate $1 / \tau$. Table 1 gives the values for these model parameters based on average arrival and activity times of active users.

User activities consist of voting, creating resolves, and forming links. User activity is clumped in time, with groups of many votes close in time separated by gaps of at least several hours. This temporal struc-

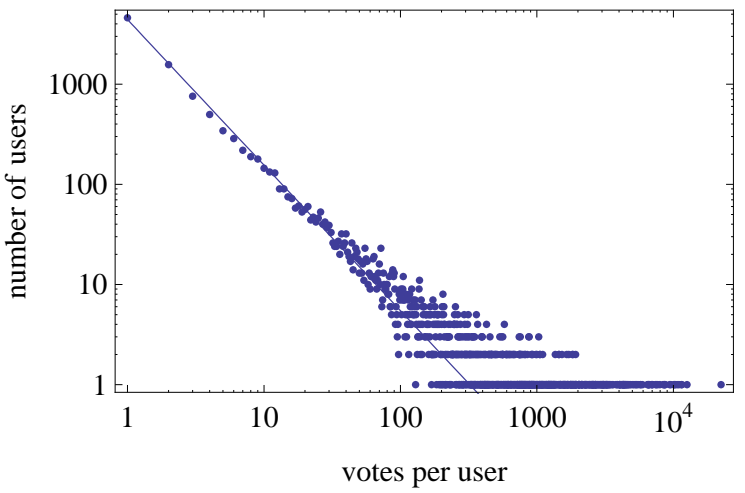

Figure 2: Distribution of number of users vs. the number of votes a user made. The solid curve indicates a Zipf distribution fit to the values, with parameter $\nu=0.45 \pm 0.01$. In this and other figures the range given with the parameter estimate is the 95\% confidence interval. The plot does not include the 2984 users with zero votes.

\begin{tabular}{ll} 
parameter & value \\
\hline new user rate & $\alpha=9.3 /$ day \\
activity time constant & $\tau=124$ days \\
resolve creation & $q=0.018 \pm 0.0002$ \\
link creation & $\lambda=0.043 \pm 0.0003$
\end{tabular}

Table 1: User activity parameters.

ture can be viewed as a sequence of user sessions. The averaged distributions for interevent times between activities of individuals show long-tail behavior, similarly to other observed human activity patterns, such as email communications or web site visits 30. To model the number of votes per user in the long time limit where we are only interested in the total number of accumulated votes for a particular user, this clumping of votes in time is not important. Specifically we suppose each user has an average activity rate $\rho$ while they are active on the site (cf. Fig. 1), given as $\rho_{u}=e_{u} / T_{u}$, where $\rho_{u}$ is user $u$ 's activity, $e_{u}$ is her number of events (i.e., votes, resolve creations and links), and $T_{u}$ is the time elapsed between her first and last vote. We suppose the $\rho_{u}$ values arise as independent choices from a distribution $P_{\text {user }}\left(\rho_{u}\right)$ and the values are independent of the length of time a user is active on the site. These properties are only weakly correlated (correlation coefficient -0.06 among active users).

We characterize user activities by fractions $q$ and $\lambda$ 


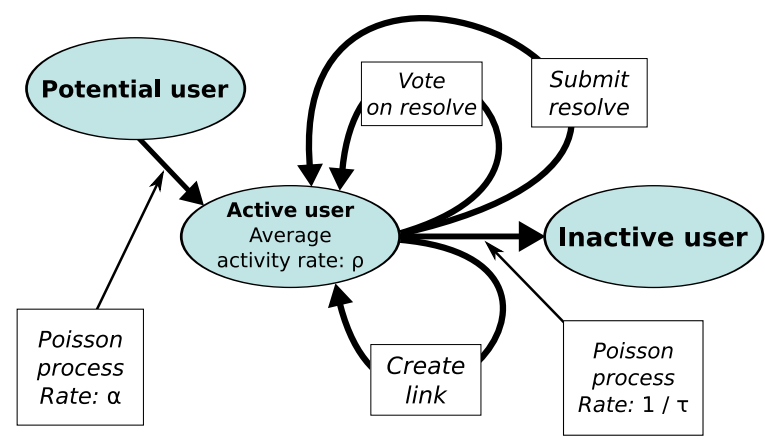

Figure 3: Model of user behavior. People join the site as active users, who create resolves, vote on them and link to other active users. Users can eventually stop participating and become inactive.

for creating resolves and forming links, respectively. The rate of voting on existing resolves for a user is then $\rho_{u}(1-q-\lambda)$, which is by far the most common of the three user activities. For simplicity, we treat these choices as independent and take $q$ and $\lambda$ to be the same for all users. Thus in our model, the variation among users is due to their differing overall activity rates $\rho_{u}$ and amount of time they are active on the site $T_{u}$.

\subsection{Behavior}

We estimate the model parameters from the observed user activities, and restrict attention to active users. Table 1 shows the estimates for parameters, $q$ and $\lambda$, governing activity choices. Fig. 4 shows the observed cumulative distribution $\rho_{u}$ values and a fit to a lognormal distribution.

The heavy tailed nature of the votes per user distribution (Fig. 2) can be attributed to the interplay between the user activity times $T_{u}$ and the broad lognormal distribution of the user activity rates $\rho_{u}$ : the mixture of these two distributions results in a power law, as has been shown in the context of web page links as well [16.

The distributions of activity times and rates presumably reflect the range of dedication of users to the site, where most users are trying the service for a very limited time but active users are also represented in the heavy tail. Such extended distributions of user activity rates is also seen in other activities, including use of web sites, e.g., Digg 22, and scientific productivity [28].

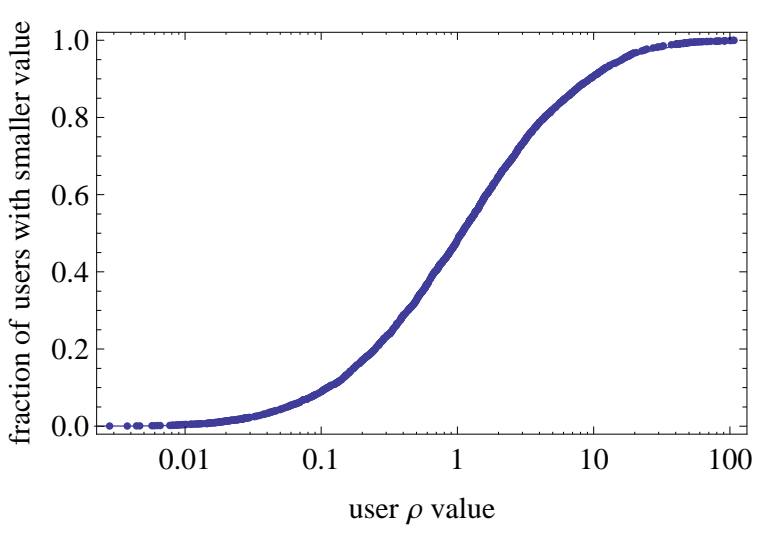

Figure 4: Cumulative distribution of activity rates, $\rho_{u}$, for the 4719 users who were active at least one day and voted on at least one noninitial resolve or formed at least on link. Plot includes a curve for a lognormal distribution fit, which is indistinguishable from the points and with parameters $\mu=0.03 \pm 0.05$ and $\sigma=1.70 \pm 0.04$. The $\rho$ values are in units of actions per day.

\section{Resolves}

A key question for user-created content is how user activities distribute among the available content. For Essembly, Fig. [5 shows the total number of votes per resolve. This distribution covers a wide range, with some resolves receiving many times as many votes as the median. In Essembly, each resolve receives its first vote when it is created, i.e., the vote of the user introducing the resolve. Thus the observed votes on a resolve are a combination of two user activities: creating a new resolve (giving the resolve its first vote) and subsequently other users choosing to vote on the resolve if they see it while visiting the site. We note that users do not see the distribution of previous votes until they cast their votes, so that their judgement is unbiased. After voting, they can see how other users had voted on the resolve.

We consider a user's selection of an existing resolve to vote on as mainly due to a combination of two factors: visibility and interestingness of a resolve to a user. Visibility is the probability a user finds the resolve during a visit to the site. Interestingness is the conditional probability a user votes on the resolve given it is visible to that user. These two factors apply to a variety of web sites, e.g., providing a description of average behavior on Digg [21]. 


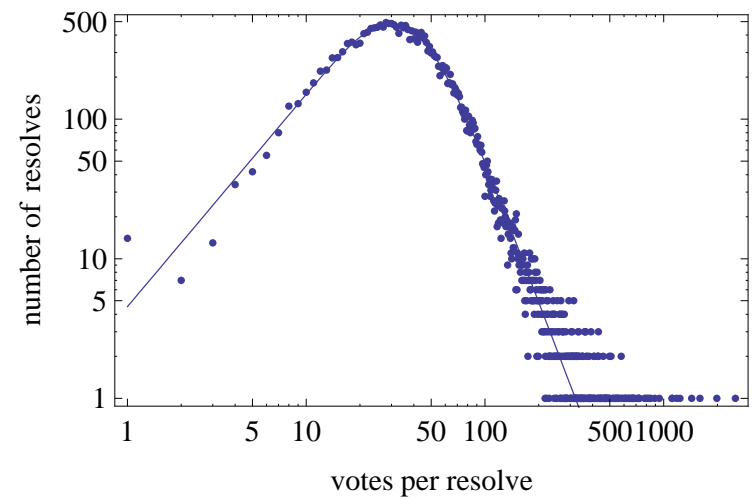

Figure 5: Distribution of votes on resolves. The solid curve indicates a double Pareto lognormal fit to the values, with parameters $\alpha=2.4 \pm 0.1, \beta=2.5 \pm 0.1$, $\mu=3.67 \pm 0.02$ and $\sigma=0.38 \pm 0.02$.

The design of the web site's user interface determines content visibility. Typically sites, including Essembly, emphasize recently created content and popular content (i.e., receiving many votes over a period of time). Essembly also emphasizes controversial resolves. As with other networking sites, the user interface highlights resolves with these properties both globally and among the user's network neighbors. Users can also find resolves through a search interface. While we cannot observe which resolves people click on, we do register when they vote on them, and thus find it interesting enough to warrant spending time to consider them.

In a similar vein, clickthrough rates have been extensively investigated in the context of web search and search engine optimization. Web search engines strive to provide users with relevant results to their queries, and rank the matching documents in reverse order of perceived importance to the searcher. However, due to the fact that search queries are not well defined and several possible optimal results may match a user's request, it is not always the top ranked result that is most relevant to the user. Search engine logs provide data on which results users click on for given queries, and thus can reveal users' implicit relevance judgements to their searches. It has been shown, however, that the probability of clicking on a given result is biased by the presentation order, thus a result with the same relevance as another but appearing in a higher poisition may get more clicks (this is also called "trust bias") [10, 9].

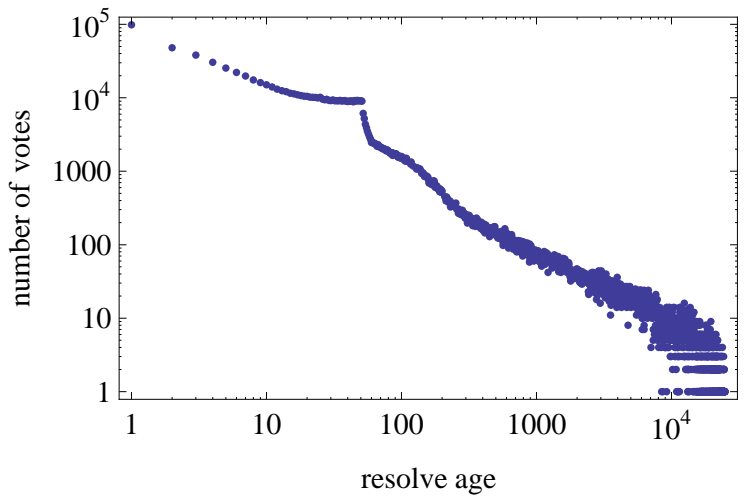

Figure 6: Distribution of votes vs. age of a resolve.

Eyetracking experiments have also shown that users scan through search results in a linear order from top to bottom, which further explains why results on the top are clicked with a larger probability [18. Clickthroughs are analogous to votes cast on resolves in Essembly, indicating a preference on the part of the user for the given item found for the query during a web search, and the resolve voted on in Essembly, respectively. Predictive models have been developed to compensate for position bias and to offset it to reveal the true relevance of the search results for the users [1, 8].

In Essembly, recency appears to be the most significant factor affecting visibility, in a very similar manner to how search engine users perceive the ranked results. Fig. [6] shows how votes distribute according to the age of the resolve at the time of the vote. We define the age of the resolve as the ordinality of the given resolve among resolves introduced in time. An age 1 resolve is the newest one of the resolves introduced, while the oldest resolve has age $R$ where $R$ is the number of resolves. Most votes go to recent resolves with a small age.

The decay in votes with age is motivated by recency (decreasing visibility with age as resolve moves down, and eventually off, the list of recent resolves). We offer no underlying model for this "aging function" but its overall power-law form corresponds to users' willingness to visit successive pages or scroll down a long list [16. The step at age 50 is, presumably, due to a limit on number of recent resolves readily accessible to users. The values decrease as a power law, proportional to $a^{-s}$, where $a$ is resolve age and $s \approx 0.5$ up to about age 50. For larger ages, the values in Fig. [6] decreases faster, with $s \approx-1$. It has 
also been found that in search engine result pages the probability of clicking on a result also decreases with the rank of the result as a power law, albeit with a different exponent $(-1.6)$ 20, 12 .

The combination of different ages in the data sample is a significant factor in producing the observed distributions [15. In particular, a distribution of ages and a multiplicative process produces a lognormal distribution with power-law tails, the double Pareto lognormal distribution [26, with four parameters. Two parameters, $\mu$ and $\sigma$ characterize the location and width of the center of the distribution. The remaining parameters characterize the tails: $\alpha$ for the power-law decay in the upper tail, with number of resolves with $v$ votes proportional to $v^{-\alpha-1}$, and $\beta$ for the power-law growth in the lower tail, with number of resolves proportional to $v^{\beta-1}$. Fig. 5 shows a fit of this distribution to the numbers of votes different resolves received. For Essembly, the networks have only a modest influence on voting [14].

\subsection{Model}

Our model of resolve creation, described in Sec. 3 involves a fraction $q$ of each user's activity on the site, on average, giving each resolve its first vote. For subsequent votes, we view a user's choice of resolve as due to an intrinsic interestingness property $r$ of each resolve and its visibility.

In general $r$ could depend on the resolve age and its popularity (especially among network neighbors, if neighbors influence a user to vote rather than just make a resolve more visible). However, for simplicity, we take $r$ to be constant for a resolve. A key motivation for this choice is the observation that high or low rates of voting on a resolve tend to persist over time, when controlling for the age and number of votes the resolve already has. Thus the importance of an intrinsic interestingness property of resolves is a reasonable approximation for Essembly (as discussed further in Sec. (6). We further assume $r$ is independent of the user, which amounts to considering general interest in resolves among the population rather than considering possible niche interests among subgroups of users. With these simplifications, we take the $r$ values to arise as independent choices from a distribution $P_{\text {resolve }}(r)$.

Visibility of a resolve depends on age, rank in number of votes compared with other resolves (popularity), controversy, both in general and among user's neighbors. For Essembly, resolve age appears to be

\begin{tabular}{c|cccc} 
& \multicolumn{4}{|c}{ interval } \\
resolve & $I_{1}$ & $I_{2}$ & $I_{3}$ & $I_{4}$ \\
\hline 1 & $f(1) r_{1} v_{1}$ & $f(2) r_{1} v_{2}$ & $f(3) r_{1} v_{3}$ & $f(4) r_{1} v_{4}$ \\
2 & - & $f(1) r_{2} v_{2}$ & $f(2) r_{2} v_{3}$ & $f(3) r_{2} v_{4}$ \\
3 & - & - & $f(1) r_{3} v_{3}$ & $f(2) r_{3} v_{4}$ \\
4 & - & - & - & $f(1) r_{4} v_{4}$
\end{tabular}

Table 2: Model of distribution of votes among resolves in time intervals between successive resolve introductions, here shown for the first four resolves.

the most significant factor, so we take visibility to be a function of age $a$ alone, as determined by a function $f(a)$.

With these factors, we model the chance that the next vote on existing resolves goes to resolve $j$ as being proportional to $r_{j} f\left(a_{j}\right)$ where $a_{j}$ is the age of the resolve at the time of the vote. The model's behavior is unchanged by an overall multiplicative constant, and we arbitrarily set $f(1)=1$.

\subsection{Behavior}

We would like to estimate the distribution $P_{\text {resolve }}$ and the aging function $f(a)$. To do so, we consider the votes (other than the first vote on each resolve) between successive resolve introductions. Specifically, let $R$ be the number of resolves in our data sample. We denote the resolves in the order they were introduced, ranging from 1 to $R$.

Let us assume that there have $i$ resolves been introduced in Essembly up to a given time, and let $v_{i}$ be the number of votes made in the time interval $I_{i}$ between the introductions of resolves $i$ and $i+1$ (not including the two votes accompanying those resolve introductions). During this interval, the system has $i$ existing resolves as assumed. When the number of existing resolves is large, we can treat the votes going to each resolve as approximately independent. In this case, the number of votes resolve $j \leq i$ receives during time interval $I_{i}$ is a Poisson process with mean $v_{i} r_{j} f(i-j+1)$ because during this interval resolve $j$ is of age $i-j+1$. Table 2 illustrates these relationships.

We estimate the $r_{j}$ and $f(a)$ values as those maximizing the likelihood of getting the observed numbers of votes on the resolves in these time intervals, coming from independent Poisson distributions. This maximization does not have a simple closed form, but setting derivatives with respect to these parameters 


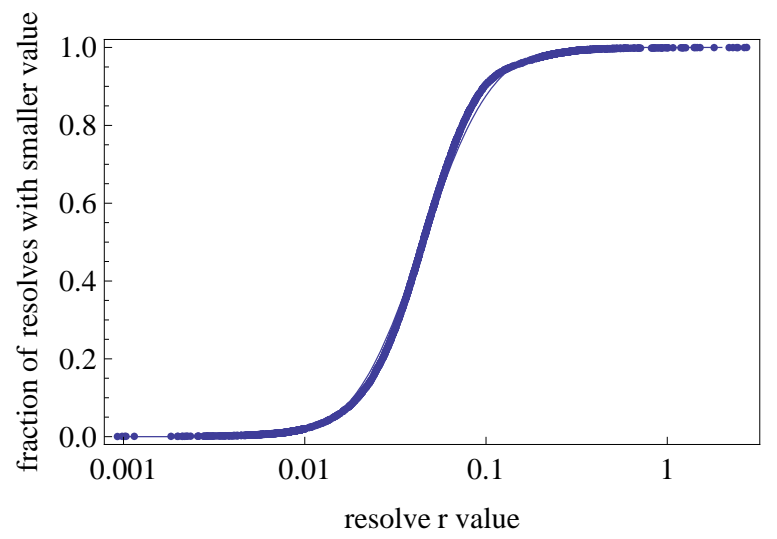

Figure 7: Cumulative distribution of $r$ values for the resolves as obtained from a maximum likelihood estimate for the observed data. The curve shows a lognormal distribution fit, with parameters $\mu=-3.11 \pm 0.01$ and $\sigma=0.69 \pm 0.01$.

to zero does give simple relations between these values at the maximum:

$$
\begin{aligned}
r_{j} & =\frac{v_{j}^{\text {resolve }}}{\sum_{a=1}^{R-j+1} f(a) v_{a+j-1}} \\
f(a) & =\frac{v_{a}^{\text {age }}}{\sum_{j=1}^{R-a+1} r_{j} v_{a+j-1}}
\end{aligned}
$$

where $v_{j}^{\text {resolve }}$ is the number of votes resolve $j$ has received, $v_{a}^{\text {age }}$ is the number of votes made to resolves of age $a$ at the time of the vote, in both cases excluding the initial vote to each resolve. The resulting $f(a)$ estimates from the numerical solution are similar to the distribution of votes vs. age in Fig. 6, and Fig. 7 shows the distribution of estimated $r$ values and a lognormal fit.

With the wide variation in $r$ values for resolves and the activity rates for users (Fig. (4), a natural question is whether these variations are related. In particular, whether the most active users tend to preferentially introduce resolves that are especially interesting to other users. While active users tend to introduce more resolves overall, the correlation between the activity rate of a user and the average $r$ values of the resolves introduced by that user is small: -0.06 . We find a modest correlation (0.16) between the time a user is active on the site and the mean $r$ values of that user's introduced resolves.

To relate this model to the vote distribution of Fig. 5 consider the votes received by resolve $j$ up to and including the time it is of age $A$. According to our model, the number of votes, other than its first vote, this resolve receives is a Poisson variable $V_{j}(A)$ with mean

$$
\mu_{j}(A)=r_{j} \sum_{a=1}^{A} f(a) v_{j+a-1}
$$

At the end of our data set, resolve $j$ is of age $R-j+1$.

The persistence of votes on resolves based on the wide variation of $r$ values among resolves gives rise to a multiplicative process with decay. To see this, in our model the number of votes between successive resolve introductions is geometrically distributed with mean $\hat{v}=(1-q-\lambda) / q \approx 52$. Furthermore, from Fig. 6. the aging function is approximately power law, with $f(a) \approx a^{-s}$ and $\sum_{a=1}^{A} f(a) \sim A^{1-s} /(1-s)$. The expected number of votes up to age $A$ is then $\mu_{j}(A) \sim r_{j} \hat{v} A^{1-s} /(1-s)$. After accumulating many votes (i.e., when $A$ is large), the actual number of votes $V_{j}(A)$ will usually be close to this expected value. The change in votes to age $A+1$ is

$$
\begin{aligned}
V_{j}(A+1) & \approx r_{j} \hat{v} \frac{A^{1-s}}{1-s}(1+x) \\
& \approx V_{j}(A)(1+x)
\end{aligned}
$$

where $x$ is a nonnegative random variable with mean $(1-s) / A$. Thus, except possibly for the votes a resolve receives shortly after its introduction, the growth in number of votes is well-described by a multiplicative process with decay.

That our model corresponds to a multiplicative process has two consequences. First, a sample obtained at a range of ages from a multiplicative process (with or without decay) leads to the double Pareto lognormal distribution seen in Fig. 5 . In our case, the sample has a uniform range of ages from 1 to $R$, though with the decay older resolves accumulate votes more slowly than younger ones. A second consequence arises from the decay as resolves become less visible over time. Thus our model provides one mechanism using locally available information giving rise to dynamics governed by multiplicative random variation with decay. A similar process arises if the decay is due to any combination of decreasing interest in the content and loss of visibility with age, e.g., as seen in sites such as Digg [31] with current events stories that become less relevant over time.

As one indication of the diversity of voting on resolves, Fig. 8 shows how the average $r$ value for resolves receiving votes compares to the average for all 


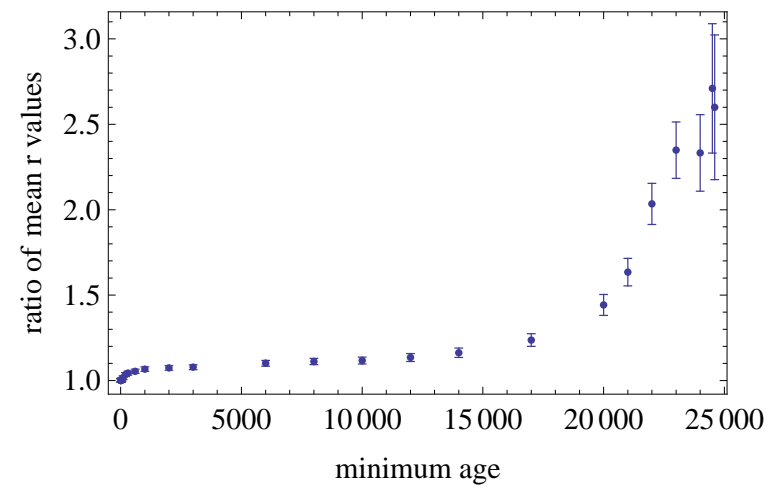

Figure 8: Ratio of means of $r$ estimates for resolves receiving votes at or after various ages to the $r$ estimates for all resolves of those ages. Error bars indicate the standard error in the ratio of means from the standard deviation of the $r$ values and the number of resolves in each category.

resolves among those at least a given age. Randomization tests indicate average $r$ values of resolves receiving votes are unlikely to be the same as those of all resolves at each of these ages, with $p$-values less than $10^{-3}$ in all cases. With increasing age, resolves continuing to receive votes tend to be those with especially high $r$ values. This behavior indicates that high interestingness estimates for resolves persist over time, as a small subset of resolves continue to collect votes well after their introduction.

\section{$5 \quad$ Links}

Users' decisions of who to link to and how they attend to the behavior of their neighbors can significantly affect the performance of participatory web sites. A common property of such networks is the wide range in numbers of links made by users, i.e., the degree distribution of the network. The structure of the networks is typical of those seen in online social networking sites, and the links created by users generally conform to their nominal semantics [14].

The degree distributions in all three Essembly networks are close to a truncated power law 25], with number of users in the network with degree $d$ proportional to $d^{-\tau} e^{-d / \kappa}$. Fig. 9 shows the distribution of degrees in the networks.

These long-tail degree distributions are often viewed as due to a preferential attachment process in which users tend to form links with others in propor-

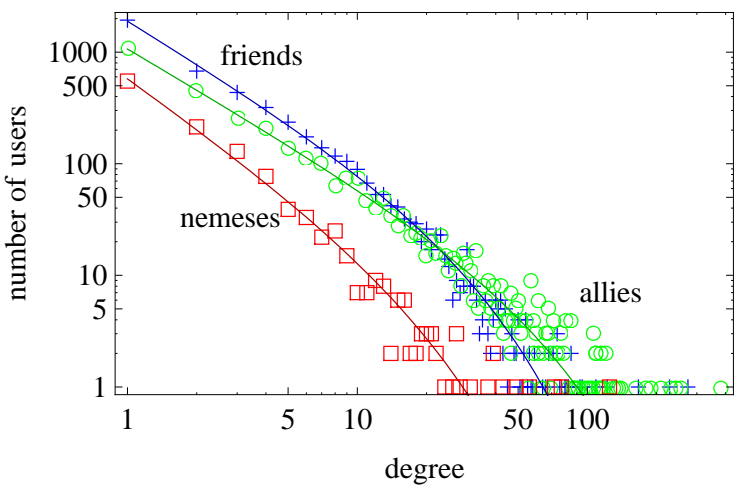

Figure 9: The number of users in the Essembly social network who have a given number of links of the indicated type (plus symbols are for the friends, circles for the allies, and squares for the nemeses networks, respectively). The parameters of the best fits of truncated power laws on the three sets of data are given in the text.

tion to how many links they already have. Combined with a limitation on the number of links a user has, this process gives truncated power-law degree distributions [2]. For Essembly, this limitation arises from users becoming inactive, since such users no longer accept links. However, users in Essembly have no direct access to number of links of other users. Thus we need to identify a mechanism users could use, based on information available to them.

The mechanism underlying preferential attachment likely differs between the friends network and the two ideological ones. In particular, since the links appear to follow their nominal semantics [14, links in the friends network are likely to be mainly between people who know each other (i.e., not found via Essembly) while ideological links (especially those who are not also friends) require finding the people by ideological profile (which Essembly makes available). Building such a profile requires voting, so a user with many votes is more likely to have voted on similar resolves as other users. Such common votes allow ideological comparisons between users and therefore suggestions for potential users to link to.

The need to build ideological profiles suggests votes on common resolves is key to the number and type of links. That is, a user forming a link is more likely to have many common votes with other users who are very active (and hence have many votes). Thus forming links based on common votes is likely to lead 
people to link preferentially to highly active users, who will in turn tend to have many links.

One challenge to evaluating this mechanism is causation: resolves voted on by network neighbors are highlighted in the user interface, making them more visible and hence more likely to receive votes. Thus common votes increase the chances of forming a link by providing information to form a profile, and links increase the chance of common votes through visibility of resolves. Separating these effects is especially challenging since our data set does not indicate when each link was formed.

We can partially address this challenge through two observations. First, Essembly presents "resolves in your network" grouping the three networks together. So any influence on resolve visibility due to networks should be similar for all networks. Second, Fig. 10 illustrates a distinction between the ideological networks in Essembly and the social network nominally linking people who know each other as friends. The figure shows friends generally have many more resolves in common, i.e., both users voted on, than random pairs of users who participate in at least one of the networks. The figure also shows the ideological networks (both allies and nemeses) are similar and have significantly more common resolves than the friends network.

These two observations suggest the enhanced number of common votes for the friends network compared to random pairs is primarily due to the increased visibility of resolves due to network neighbors voting on them. Because Essembly presents resolves from all networks together, this enhancement is also likely to be the same for the ideological networks. Hence, the remaining increase in common votes in the ideological networks compared to the friends network suggests the additional commonality required for users to form the links.

Fig. 11 shows the types of links vary depending on user activity. For this plot, users with at least one network connection are grouped into quantiles by their number of votes. Each point on the plot is the average fraction of link types among users in that quantile, with the error bar indicating the standard deviation of this estimate of the mean. Users with few votes tend to have most of their links to friends only, so do not participate much in the ideological networks. On the other hand, users with many votes tend to have most of their links in the ideological networks and to people who are not also friends. The same trend in link types occurs as a function of other

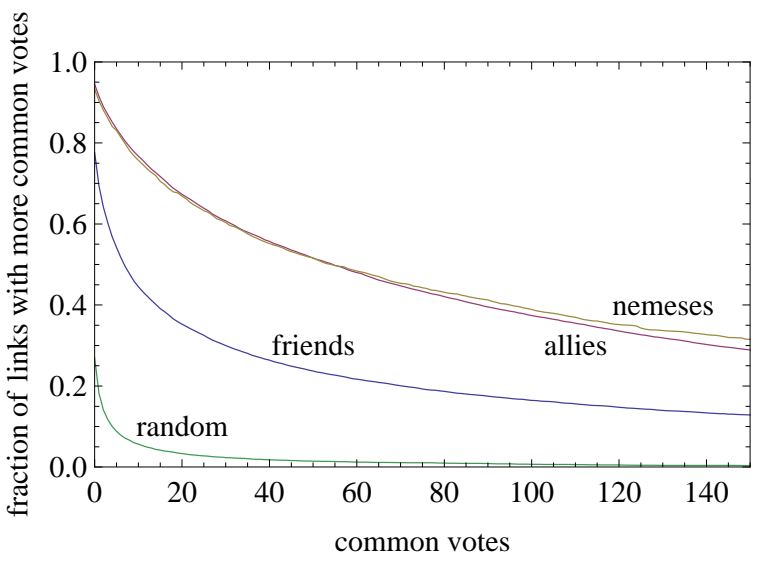

Figure 10: Cumulative distribution of number of common votes among linked pairs in the networks, and among random pairs of users who are in at least one network. For each number of common votes, the curves show the fraction of pairs with more than that many resolves both users in the pair voted on.

measures of user activity, i.e., using quantiles based on the time a user is active or the number of links a user has.

\subsection{Model}

In our model, user $u$ forms links at a rate $\lambda \rho_{u}$. Thus the number of links a user forms is a combination of activity rate and how long the user remains at the site. The wide variation in activity times and $\rho_{u}$ among users (Fig. [1 and 4) gives rise to a wide distribution of number of links.

While the most common mechanisms designed to reproduce the observed power law degree distributions use growing rules and the degree of vertices in link formation [11, in the following we propose a mechanism that only takes into account the extent to which two users share interests to describe link formation between two users.

Because links involve two people, an additional modeling issue is which pairs of users form links. In our model, we take the friends network to primarily reflect a preexisting social network. For the ideological networks, however, we take the choices to depend on common votes. Furthermore, only active users can form links. Specifically, we model the likelihood a (non-friend) pair forms a link in an ideological network as proportional to the number of common votes they have. In addition, existing friends links can add 


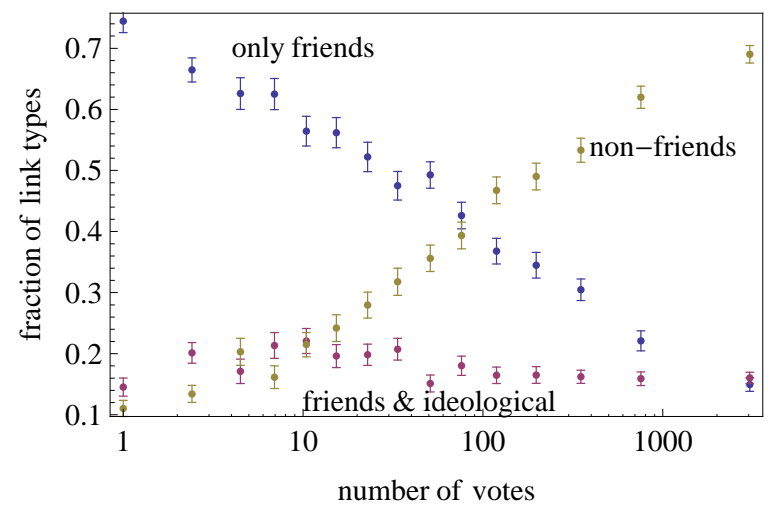

Figure 11: Fraction of link types vs. number of votes on noninitial resolves. A linked pair of users are denoted "only friends" when their only link is in the friends network, "non-friends" when they are not linked in the friends network, and "friends \& ideological" when they have a friends link as well as a link in the allies or nemeses networks.

an ideological link.

People form ideological links based on common votes, and only users active at the same time can form links. The first factor gives more links for those who vote a lot (due to being more likely to have votes in common with others). This leads to, in effect, preferential attachment for forming links (those with more links are likely to be users with more votes, hence more overlap with others), while the attachment probability does not explicitly depend on degrees. The activity constraint limits the link growth, corresponding to descriptive models giving truncated power-law degree distribution [2].

\subsection{Behavior}

To verify whether users connect to each other based on similarities in their voting profile, we propose the following simplified mechanism for link formation. Suppose that user $A$ voted on $N_{A}$ resolves, while user $B$ voted on $N_{B}$ resolves in total. Assuming that $A$ and $B$ form a link with a probability proportional to the number of votes that the pair has in common, this probability will be $P_{l}(A, B) \propto N_{A} N_{B}$ if $N_{A}$ and $N_{B}$ are sufficiently smaller than the number of all resolves, and $A$ and $B$ vote independently of each other and pick resolves randomly from the pool of all available resolves. Caldarelli et al. have shown that if vertices in a network possess intrinsic "fitnesses", and the linking probability is proportional to the product of fitnesses of the two vertices to be linked, then in the particular case when the fitnesses are drawn from a power-law probability distribution function the resulting degree distribution will have the same exponent as the fitness distribution [7]. We can consider the number of votes a person makes as the fitness of the vertex, and arrive by analogy at the same model as Ref. [7, resulting in an expected power-law exponent of -1.45 (Fig. 22).

Fitting truncated power laws to the degree distributions of the three networks shown in Fig. 9, we found the parameters $\tau_{F}=1.25 \pm 0.04, \kappa_{F}=27 \pm 4$; $\tau_{A}=1.20 \pm 0.04, \kappa_{A}=59 \pm 9$; and $\tau_{N}=1.44 \pm 0.11$, $\kappa_{N}=18 \pm 6$ for the friends, allies, and nemeses networks, respectively, with the values for the $95 \%$ confidence intervals indicated. The power-law exponents are in the range $[1.20,1.44]$, giving a consistent match to the exponent of Fig. 2. The truncation of the power laws seen in the degree distributions are most likely the result of vertices gradually becoming inactive in time. An interesting consequence of the above is that while the friends network as seen on Essembly is supposed to not be a result of shared votes made conspicuous by the web user interface, we see a consistent match in the exponents: this suggests that friendship links in real life may also form around shared interests, and that the scope of interests people have may follow a similar probability distribution function as shown in Fig. 2,

Unlike random graph models with this degree distribution [25], our mechanism based on common votes also gives significant transitivity, comparable to that observed for the allies network. That is, if users $A$ and $B$ have voted on many resolves in common, as have users $B$ and $C$, then users $A$ and $C$ also tend to have significant overlap in the resolves they voted on.

A further consequence of our model with ideological links depending on common votes is the prediction of a change in the types of links users make as they vote. In particular, users with few votes will also have few common votes with other users and hence their links will tend to be mostly friends. Users with many votes, on the other hand, will tend to have common votes with many others and hence, according to this model, tend to have mostly ideological links. This change in type of links as a function of a user's number of votes or links occurs in Essembly, as seen in Fig. 11]

Finally, our model also describes the significant fraction of users who form no links as due to a combi- 


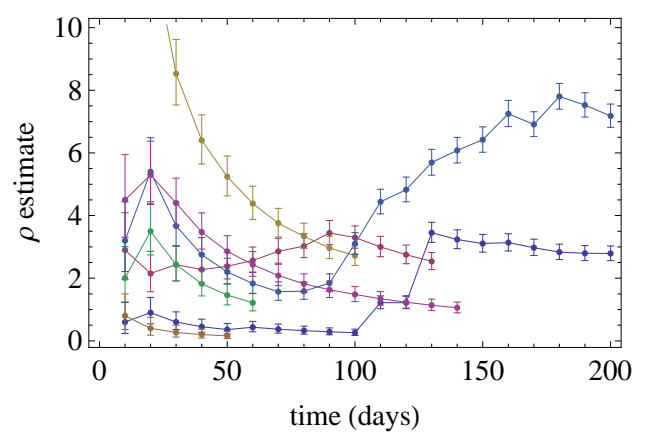

Figure 12: Estimates of $\rho$ values for several users as a function of the time since their first vote. Error bars show the $95 \%$ confidence intervals.

nation of low activity rate $\rho$ and short activity time $t$. Specifically, in our model the probability a user has no links is $e^{-\lambda \rho t}$. For active users, whose activity time distribution is roughly exponential with time constant $\tau$, the values in Table 1 and the distribution of $\rho$ values in Fig. 4 give the probability for no links as the average value of $e^{-\lambda \rho t}$ equal to $23 \%$. This compares with the 1242 out of 4762 active users (i.e., $26 \%$ ) who have no links in our data set.

\section{Online Estimation}

Our model allows estimating parameters for new users and new resolves as they act in the system. In particular, we describe using the early history of resolves to estimate the number of votes a resolve will eventually have as well as which resolve will likely receive the next vote.

Fig. 12 shows estimates of user activity levels as a function of time since the user first voted. We see user activity levels change with time, and in different ways. So users not only differ considerably in their average activity rates but also in how their interest in the site varies in time.

For resolves, using the model of Sec. 4. Fig. 13 shows how estimates for resolves, and their confidence intervals change over time, as more votes are observed. Other resolves show similar behavior. Thus the interestingness of resolves appears to converge in time as we expect.

In practice, however, the optimization procedure is computationally very costly due to the large number of parameters that grows linearly with the number of resolves in the system. A further requirement of an online algorithm is that it is able to update the

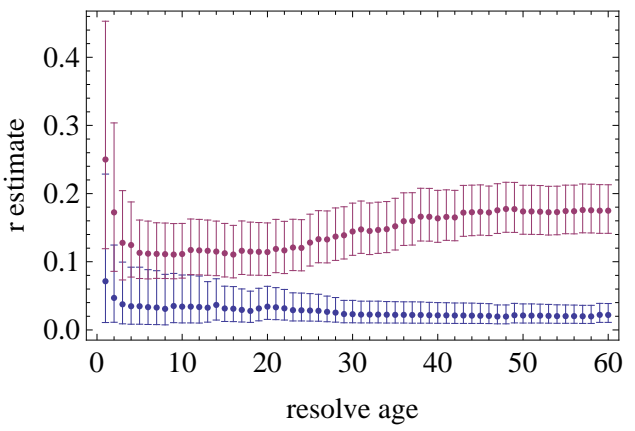

Figure 13: Estimates of $r$ values for two resolves as a function of their age. Error bars show the $95 \%$ confidence intervals.

model parameters in real time as new users, votes and resolves enter the system. Thus it is not feasible to consider a growing number of resolves with constant resources. Instead we must limit the the number of parameters and thus resolves to be optimized to a constant value.

One such approach is to optimize parameters based on the last $K$ active resolves only, and keep the interestingness and aging parameters constant for resolves older than that. This method, interestingly, has the potential benefit of being able to track changes to interestingness and aging in time.

Another incremental approach uses the observation that old resolves, with a long track record of votes, have their interestingness well-estimated and similarly the aging function $f(a)$ for small ages is wellestimated from prior experience with many resolves receiving votes at those ages. Conversely, recently introduced resolves have had little time to accumulate votes and $f(a)$ for large ages is poorly estimated due to having little experience in the system with resolves that old. Furthermore, we can expect $f(a)$ to change slowly with time as primarily due to how the user interface makes resolves visible to users. The maximum likelihood estimation for these parameters described in Sec. 4 requires a computationally expensive optimization to find the best choices for $r_{j}$ and $f(a)$ for all values. For new resolves, with $j$ close to $R$, Eq. (11) determines the $r_{j}$ values in terms of the values of $f(a)$ for small ages (i.e., $a=1, \ldots, R-j+1$ ) which are already well-determined from the prior history of the system. So instead of an expensive reevaluation of all the $r$ and $f$ values, we can simply incrementally estimate the $r$ values of new resolves assuming $f(a)$ values for small ages do not change much. Conversely, 
as new resolves are introduced, the oldest resolves in the system advance to ever larger ages, allowing estimates of $f(a)$ for those ages from Eq. (2) by assuming the $r$ values of those old resolves do not change much with the introduction of new resolves.

Such estimates of model parameters can be useful guides for improving social web sites if extended to user behavior as well, by identifying new users likely to become highly active or content likely to become popular. Since it is possible to estimate the statistical errors given the sample size, one can also perform risk assessment when giving the estimates. Newly posted content with high interestingness, for instance, can be quickly identified and given prominent attention on the online interface.

\section{Discussion}

We described several extended distributions resulting from user behavior on Essembly, a web site where users create and rate content as well as form networks. These distributions are common in participatory web sites. From the extended distributions of user behavior we find extremely heterogenous population of users and resolves. We introduce a plausible mechanism describing user behavior based on locally available information, involving a combination of aging and a large variation among people and resolves. We centered our investigations around three areas: the wide range in user activity levels in online participation; how online social networks form around topical interests; and the factors that influence the popularity of user-created content.

In particular, we found, first, that most users try the online services only briefly, so most of the activity arises from a relatively small fraction of users who account for the diverse behavior observed. Second, we gave a plausible, quantitative explanation of the longtailed degree distributions observed in online communities, based on only the observed activity patterns of users and the underlying collaborative mechanisms. Our observations suggest different mechanisms underly the formation of the social (friends) and ideological (allies and nemeses) networks, although these mechanisms give similar outcomes, e.g., for the qualitative form of the degree distribution. The implications may extend beyond the scope of purely online societies to describe other societal connections as well where shared interests motivate relationship formation. Our model, however, does not address other significant properties of the networks, such as community structure and assortativity and why they differ among the three networks [14]. Nor does our model address detailed effects on user behavior due to their network neighbors. Third, we proposed a model and algorithm that can describe and predict through iterative refinements how the popularity of user-generated submissions evolves in time, considering both their changing exposure online and their inherent interestingness. We found that the exposure that content receives depends largely on its recency, and decays with age.

The characteristics of our models plausibly apply to other web sites where user participation is selfdirected and where content creation and social link formation plays a dominant part in the individual online activities. The Digg and Wikipedia user communities (those whose activity data is publicly available) in particular may show similar behavior in their activity patterns. Our models could be extended to include the weak, but nevertheless statistically significant, correlations among user behaviors such as activity rate and the time they remain active on the site. Including such correlations, as well as some historical and demographic information on individual users, may improve the model predictions as seen, for example, in models estimating customer purchase activities [6].

Consequences of our model include suggestions for identifying active users and interesting resolves early in their history. E.g., from persistence in voting rates over time, even before accumulating enough votes to be rated as popular. Such identification could be useful to promote interesting content on the web site more rapidly, particularly in the case of niche interests. Beyond helping users find interesting content, designs informed by causal models could also help with derivative applications, such as collaborative filtering or developing trust and reputations, by quickly focusing on the most significant users or items. Such applications raise significant questions of the relevant time scales. That is, observed behavior is noisy, so there is a tradeoff between using a long time to accumulate enough statistics to calibrate the model vs. using a short time to allow responsiveness faster than other proxies for user interest such as popularity.

Our models raise additional questions on population properties we used. One such question is understanding how the resolve aging function relates to the user interface and changing interests among the user population. Another question is how the wide distri- 
butions in user activity and resolve interestingness arise. The lognormal fits suggest underlying multiplicative processes are involved. It would also be interesting to extend the model to identify niche resolves, if any. That is, resolves of high interest to small subgroups of users but not to the population as a whole. Automatically identifying such subgroups could help people find others with similar interests by supplementing comparisons based on ideological profiles.

A caveat on our results, as with other observational studies of web behavior, is the evidence for mechanisms is based on correlations in observations. While mechanisms proposed here are plausible causal explanations since they rely on information and actions available to users, intervention experiments would give more confidence in distinguishing correlation from causal relationships. Our model provides testable hypotheses for such experiments. For example, if intrinsic interest in resolves is a major factor in users' selection of resolves, then deliberate changes in the number of votes may change visibility but will not affect interestingness. In that case, we would expect subsequent votes to return to the original trend. Thus one area for experimentation is to determine how users value content on various web sites. For example, if items are valued mainly because others value them (e.g., fashion items) then observed votes would cause rather than just reflect high value. In such cases, random initial variations in ratings would be amplified, and show very different results if repeated or tried on separate subgroups of the population. If items all have similar values and difference mainly due to visibility, e.g., recency or popularity, then we would expect votes due to rank order of votes (e.g., whether item is most popular) rather than absolute number of votes. If items have broad intrinsic value, then voting would show persistence over time and similar outcomes for independent subgroups. It would also be useful to identify aspects of the model that could be tested in small groups, thereby allowing detailed and well-controlled laboratory experiments comparing multiple interventions. Larger scale experiments [4, 27. would also be useful to determine the generality of these mechanisms.

The key features of continual arrival of new users, existing users becoming inactive and a wide range of activity levels among the user population and interest in the content can apply in many contexts. For the distribution of how user rate content (e.g., votes on resolves in Essembly), generalizing to other situa- tions will depend on the origin of perceived value to the users. At one extreme, which seems to apply to Essembly, the resolves themselves have a wide range of appeal to the user population, leading some items to consistently collect ratings at higher rates than others. At the other extreme, perceived value could be largely driven by popularity among the users, or subgroups of users, as seen in some cultural products 27. In rapidly changing situations, e.g., current news events or blog posts, recency is important not only in providing visibility through the system's user interface, but also determining the level of interest. In other situations, the level of interest in the items changes slowly, if at all, as appears to be the case for resolves in Essembly concerning broad political questions such as the benefits of free trade. All these situations can lead to long-tail distributions through a combination of a "rich get richer" multiplicative process and decay with age. But these situations have different underlying causal mechanisms and hence different implications for how changes in the site will affect user behavior. Thus, design and evaluation of participatory web sites can benefit from the availability of causal models.

\section{Acknowledgments}

We thank Chris Chan and Jimmy Kittiyachavalit of Essembly for their help in accessing the Essembly data. We have benefited from discussions with Michael Brzozowski, Dennis Wilkinson, and Tamás Sarlós.

\section{References}

[1] E. Agichtein, E. Brill, S. Dumais, and R. Ragno. Learning user interaction models for predicting web search result preferences. In Proc. of the International ACM SIGIR Conference on Research and Development in Information Retrieval, pages 3-10, 2006.

[2] L. A. N. Amaral, A. Scala, M. Barthelemy, and H. E. Stanley. Classes of small-world networks. Proc. of the Natl. Acad. Sci., 97:11149-11152, 2000.

[3] C. Anderson. The Long Tail: Why the Future of Business is Selling Less of More. Hyperion, 2006.

[4] W. S. Bainbridge. The scientific research potential of virtual worlds. Science, 317:472-476, 2007.

[5] S. Boccaletti, V. Latora, Y. Moreno, M. Chavez, and D.-U. Hwang. Complex networks: Structure and dynamics. Physics Reports, 424:157-308, 2006. 
[6] S. Borle, S. S. Singh, and D. C. Jain. Customer lifetime value measurement. Management Science, 54:100-112, 2008.

[7] G. Caldarelli, A. Capocci, P. De Los Rios, and M. A. Munoz. Scale-free networks from varying vertex intrinsic fitness. Physical Review Letters, 89:258702, 2002.

[8] B. Carterette and R. Jones. Evaluating search engines by modeling the relationship between relevance and clicks. In J. Platt et al., editors, Advances in Neural Information Processing Systems. NIPS, 2007.

[9] C. L. A. Clarke, E. Agichtein, S. Dumais, and R. W. White. The influence of caption features on clickthrough patterns in web search. In Proc. of the International ACM SIGIR Conference on Research and Development in Information Retrieval, pages 135142, 2007.

[10] N. Craswell, O. Zoeter, M. Taylor, and B. Ramsey. An experimental comparison of click position-bias models. In Proc. of the International Conference on Web Search and Web Data Mining, pages 87-94, NY, 2008. ACM.

[11] S. N. Dorogovtsev and J. F. F. Mendes. Evolution of networks. Advances In Physics, 51:1079-1187, 2002.

[12] S. Fortunato, A. Flammini, F. Menczer, and A. Vespignani. Topical interests and the mitigation of search engine bias. Proc. of the Natl. Acad. of Sciences, 103:12684-12689, 2006.

[13] R. Guha, R. Kumar, P. Raghavan, and A. Tomkins. Propagation of trust and distrust. In Proc. of the 13th Intl. World Wide Web Conf. (WWW2004), pages 403-412, New York, 2004. ACM.

[14] T. Hogg, D. M. Wilkinson, G. Szabo, and M. Brzozowski. Multiple relationship types in online communities and social networks. In Proc. of the AAAI Symposium on Social Information Processing, 2008.

[15] B. A. Huberman and L. A. Adamic. Growth dynamics of the World Wide Web. Nature, 401:131, 1999.

[16] B. A. Huberman, P. L. T. Pirolli, J. E. Pitkow, and R. M. Lukose. Strong regularities in World Wide Web surfing. Science, 280:95-97, 1998.

[17] A. James and M. J. Plank. On fitting power laws to ecological data. arxiv.org preprint 0712.0613, 2007.

[18] T. Joachims, L. Granka, B. Pan, H. Hembrooke, and G. Gay. Accurately interpreting clickthrough data as implicit feedback. In Proc. of the International ACM SIGIR Conference on Research and Development in Information Retrieval, pages 154-161, 2005.

[19] C. Lam. SNACK: incorporating social network information in automated collaborative filtering. In Proc. of the 5th ACM Conference on Electronic Commerce (EC'04), pages 254-255. ACM Press, 2004.
[20] R. Lempel and S. Moran. Predictive caching and prefetching of query results in search engines. In Proc. of the International Conference on World Wide Web, pages 19-28, 2003.

[21] K. Lerman. Social information processing in social news aggregation. arxiv.org preprint cs.cy/0703087. 2007.

[22] K. Lerman. User participation in social media: Digg study. In IEEE/WIC/ACM Intl. Conf. on Web Intelligence and Intelligent Agent Technology, pages 255-258, 2007.

[23] M. Mitzenmacher. A brief history of generative models for power law and lognormal distributions. Internet Mathematics, 1:226-251, 2004.

[24] M. E. J. Newman. Power laws, Pareto distributions and Zipf's law. Contemporary Physics, 46:323-351, 2005.

[25] M. E. J. Newman, S. H. Strogatz, and D. J. Watts. Random graphs with arbitrary degree distributions and their applications. Physical Review E, 64:026118, 2001.

[26] W. J. Reed and M. Jorgensen. The double Paretolognormal distribution: A new parametric model for size distributions. Communications in Statistics: Theory and Methods, 33:1733-1753, 2004.

[27] M. J. Salganik, P. S. Dodds, and D. J. Watts. Experimental study of inequality and unpredictability in an artificial cultural market. Science, 311:854-856, 2006.

[28] W. Shockley. On the statistics of individual variations of productivity in research laboratories. Proc. of the IRE, 45:279-290, 1957.

[29] A. Vázquez. Growing network with local rules: Preferential attachment, clustering hierarchy, and degree correlations. Physical Review E, 67:056104, 2003.

[30] A. Vázquez, J. G. Oliveira, Z. Dezso, K.-I. Goh, I. Kondor, and A.-L. Barabasi. Modeling bursts and heavy tails in human dynamics. Physical Review E, 73:036127, 2006.

[31] F. Wu and B. A. Huberman. Novelty and collective attention. Proc. of the Natl. Acad. Sci., 104:17599 17601, 2007. 